\title{
Plants, Functions and Processes Adapt to Different Environments
}

\author{
Monica Butnariu* \\ Banat's University of Agricultural Sciences and Veterinary Medicine "Regele Mihai I al Romanei" Timisoara, Romania
}

Keywords: Self-regulation; Coordination; Integration; Adaptation

\section{Executive Summary}

Bio processes that provide interactive relationships between plant organs are associated with self-regulation functions and maintaining a dynamic balance. The adaptation process is achieved indissoluble unity of the plant with the environment. Water connects all parts of the plant beginning with molecules and ending with tissues and organs, in one whole; appears to be the only substance in the plant, which suitably may perform the functions of regulating the growth, metabolic activity and controlling integration structure and functions at all levels of the organization. The main cause of impaired functional integrity during drought conditions is disruption of the internal liquid medium, donor-acceptor relations and change water gradients between organs, inhibition of metabolic reactions and biosynthesis. In drought conditions water has central role in coordinating and maintaining the integrity functions.

\section{General Remarks}

Drought, water shortage in soil and /or atmospheric temperature and high irradiation is most alarming factor limiting crop productivity and stability throughout world. Plants are affected when drought overlaps fund environmental pollution by harmful substances, salinity or cold weather. Study of functioning of adjustment of metabolic processes, plant growth and development under adverse conditions is a cardinal point in knowledge of plants to drought tolerance mechanisms that could serve as a basis for developing routing processes directed to functional status and optimization of production process. Problem assessment and adaptation mechanisms that provide plant resistance to adverse conditions and is considered a perpetual maximum value for both contemporary biological science and future of humanity.

Researches reveal peculiarities explaining adaptation and drought resistance in plants of spontaneous basis. Characteristics relate to the development and shape of the plant, the number and depth of penetration of root system, xylem and phloem properties [1]; use and storage of assimilate reserves. There are great progress in understanding of perception and signalling mechanisms of water scarcity and genes involved in plant response to drought. Investigations carried out are focused on study of mechanisms of resistance, which is achieved at molecular and cellular levels, while elucidating principles of regulating at the level of plant resistance remains less addressed. It highlights several strategies to adapt to adverse conditions: one occurs at the cellular level and includes accommodation macromolecules and micro environment in which they operate, other-level plant, and reflects integrity of the inferior hierarchical components. As reported above, most of investigations are focused on highlighting deployment of first mechanisms of adaptation strategies and tolerance, while second remains the least addressed [2]. But an environmental change plant perceives the plant upright and resistant to stressogenic factors were studied is due to the retention of functional integrity of all organs [3]. The different environmental conditions (drought, heat, salinity, flood, cooling, etc.) dehydration is first signal that induces the plant's response to abiotic stress. The concepts of integrity (completeness) express the coordinated adjustment and unification of structure and function in an activity related adaptive plant as one whole [4]. The result of functions integrity-absorption and water use, mineral nutrition, photosynthesis, respiration, growth and development, adaptation is achieved full potential productivity of vegetable organism. Nature of integrity functions is reflected by our law additively: union of the two sides appears organizational qualitatively new results greater than sum of parts, $\Sigma 1+1>2$. Integration leads to the union of simpler systems in complex systems and complex adaptive training results for the plants to survive in harsh environments.

Bioprocesses that provide coordinated active interrelations between organs are associated with self-regulation functions and maintaining a dynamic balance of these oscillations depending on environmental conditions. The action of unfavourable factors herb acts as a unified whole. The adaptation process is achieved indissoluble unity of plant with the environment. Elucidating the internal factors involved in the integration and coordination functions at the molecular, cellular, organ and plant is one of the fundamental problems of physiology compass plant. In drought conditions the main cause of impaired functional integrity of the liquid medium is disrupting internal donor-acceptor relations and change water gradients between organs, inhibition of metabolic reactions and biosynthesis [5]. In drought conditions water has a central role in coordinating and maintaining the integrity functions. Water connects all parts of the plant, and ending with molecules from tissues and organs, in one whole. The plant water forms a continuous medium with water continuously to surface of the soil and transition of liquid in the gas phase, where it evaporates from the leaves. Reduce soil water potential plants react instantly by changing its status line throughout the plant, which in turn affect metabolic reactions and interactive relationships between organs. Water the plant performs three functions: it is the mediator of effects of environment on growth and metabolic activity, acts as a growth regulator proportional to different parts of the plant; condition integrating growth and metabolic activity at cellular level. Water seems to be the only substance in the plant that can perform this function adequately triple: growth regulation, regulating metabolic activity and integration functions of the plant [6]. All other mechanisms to control and integrate growth and development are dependent on the ability of water to regulate the growth and metabolic activity.

The significance of water bioprocesses for the integration of the structure and function as well as self-regulation of the physiological processes at the molecular, cell, and plant, seen in the fact that for the

*Corresponding author: Butnariu M, Banat's University of Agricultural Sciences and Veterinary Medicine "Regele Mihai I al Romanei" Timisoara, Romania. 300645, Calea Aradului 119, Timis, Romania, Tel: +40-0-256-277; E-mail: monicabutnariu@yahoo.com

Received December 20, 2014; Accepted February 17, 2015; Published February 21, 2015

Citation: Butnariu M (2015) Plants, Functions and Processes Adapt to Different Environments. J Bioequiv Availab 7: e62. doi:10.4172/jbb.10000e62

Copyright: ( 2015 Butnariu M. This is an open-access article distributed under the terms of the Creative Commons Attribution License, which permits unrestricted use, distribution, and reproduction in any medium, provided the original author and source are credited. 
normal completion of all metabolic reactions, including the activity of the enzymes and the maintenance of the structure, it will take some degree of hydration. The result of integration and self-regulation function is homeostasis maintained plant physiological activity using endogenous control systems and is performed at all levels of organization. At molecular level, chemical and electronic interaction between cells, tissues and organs is achieved with various compoundsregulators: enzymes, vitamins, hormones, inhibitors, minerals and not least, with participation of water. It participates in maintaining cell structure by participating in the composition of protein molecules causing their conformation. Integrating functions at the cellular level is achieved by regulating the interaction of enzyme systems, membrane and genetics and the core is centre of coordinating all vital processes occurring at cellular level. Enzymes product nucleus activity catalyses biochemical reactions in plant, conditioning deployment, coordination and self-regulation reactions by which plant metabolic bioprocesses. Metabolic mechanisms of tolerance to dehydration leads to an abundance of transcripts of many genes in bioprocesses osmotic adjustment, photosynthesis, respiration, detoxification of active oxygen species, etc. [7]. The loss of the drought to reduced film hydration of protein molecules from cytoplasm, including the function of enzyme, and inactivation of enzymes, the reduction of RNA synthesis due to the inhibition of the synthesis process itself and activation ribonucleases. On the other hand, the dehydration of tissues up to a certain critical threshold, genotype-specific, enhances formation of reactive oxygen species effect on state membranes of lipid peroxide oxidation, enzyme activity, and gene device, with negative consequences for membrane permeability, degradation of proteins and nucleic acids [8]. Thanks to functioning of protective antioxidant systems in cells kept under normal dynamic balance training and liquidation processes of reactive oxygen species. Resistance to stress caused by drought, is distinctly correlated with self-owned plant tissue hydration degree. Currently it stipulates that basis for the functional integrity of plant lies at the exchange of substances or energy, organic compounds and mineral movement being ensured by special hormones [9]. Factors for integrate different bodies of donor-acceptor system serving plant, the vascular system through phloem and xylem transport, which are the driving forces of hydrostatic pressure gradients, water and osmotic potential. Disruption of correlative links physiological processes in plants can be caused by different sensitivity or predominantly affecting organs of that plant on which action is oriented factor stressogenic factors were studied. It has been shown that unfavourable action of external factors induce changes in all vital organs, which proves the existence of links correlative coordination and integration of physiological processes in organism level. Some plant species, in particular, that are distinguished by morphological characters of root system, involves certain strategies to avoid impairment caused by drought and ensure coordination of whole plant level hormones [10]. When the preserve functional integrity is achieved at plant by: stomata closure and reduced water loss through sweating, inhibition of shoot growth and reduce surface evaporation, enhancing active uptake of water by root system, control water absorption and her's status plant by increasing and deepening roots in deep soil layers and / or hydraulic conductivity. The average humidity fluctuating plant survivals depends on the effectiveness of communication relations of root and shoot. The root is the first sensor and water potential changes as chemical parameters of soil, they perceive signals from shoot and regulate growth and the internal aqueous coordinated in line with changes in external environment, contribute to mutual integration of nutrition in all metabolic reactions underlying the growth and development of organism.

The root dry conditions plays a key role in perception of soil water deficit and transmitting signals in shoot water stress [11]. Hydraulic root signal transmission to leaves is one of the main links in adaptive adjustment system of self-regulation, coordination of physiological processes and in maintaining functional integrity. The easiest way for interaction between bodies is achieved through movement of water and nutrients. Plant roots and other organs depend on flow heterotrophic assimilates formed leaves.

Shoots require water, mineral nutrients, absorb from soil, and a number of organic compounds synthesized in roots. In shoot-root interaction complex adaptive forms of property because coordinating plant of physiological processes and maintain morphological and functional integrity. There is a close correlation between the ability of roots to absorb water in drought conditions and dry mass of shoots. Successful coordination between interrelations of these two partners is complicated and many aspects of these interrelations remain unresolved. Under optimum conditions the relationship between shoot and root installs a balance that ensures optimal growth of both organs. Unlike shoot growth, root growth at early stages of moderate water deficit remains as a result of rapid osmotic adjustment, which partially stabilizes the cell wall elasticity [12]. But for osmotic adjustment and root apex continued growth is highly dependent on imports of organic osmotic compounds and leaf metabolic bioprocesses. In contrast to roots, leaf growth is inhibited already at a moderate drought. These changes are associated with changes in $\mathrm{pH}$ and inhibition of cell wall acidification, $\mathrm{H}(+)$-ATPase inactivation plasmalemma with the change of cell wall peroxidases, etc. There is a correlation between activity of GwPX and intensity of negative growth in leaves and roots of plants exposed to drought [13,14]. So, leaves and roots-places most intense exchange of energy and matter with the environment, to maintain functions require interconnection in a manner that would allow the integration of physiological plant level as a single whole. The leaves, which provides photosynthesis and $95 \%$ or more of the total water consumption are distant away from the source of water in soil, but linked to it via transport through the xylem of roots and absorptive surface. The tissue that connects these distant regions as growth and acquisitions has a key role in ensuring coordination, allowing suggesting the idea that xylem works not only as carriers of the masses, but also as a communication system and integration. Vascular system plays an role in integrating information from distant organs located by delivering not only water, inorganic nutrients and assimilates and plant hormones and chemical signals, with involvement in processes of distribution [15]. Water flow induced by sweating, has a true significance as a force of attraction and movement of mineral nutrients in soil and vice versa, their pumping plant creates an osmotic force favoring water uptake from soil. Under normal water transfer and have early in their penetration and water movement through parenchyma tissue, predominantly in young roots, the epidermis, cortex and stele root endoderm, the result through the apoplast, symplast and transcellular route across plant. Apoplast include intercellular spaces, channels of microfibrils of cellulose cell walls and xylem vessels. The upward flow of water through apoplast generally takes place in xylem vessels (90-99\%) of the fibrils and pores of cell wall and intercellular spaces from the roots to the leaves (1-10\%). Symplast continuum cytoplasm of all cells is interconnected by plasmodesmata in a single system. And finally, transmembrane pathway is transfer of water from cell to cell to exit vacuole and plasma membrane into intercellular space. Empirically there cannot be separate transfer path by symplast from way transcellular route, so these two paths were summarily called "transfer of cell-to-cell" [16]. The proportion of water movement in various ways depending on the driving forces involved in transporting 
them. The importance of water movement in cell-to-cell increase under the perspiration is blocked.

Potential regulators of water movement radially and axially from roots to leaves are aquaporine, hollow xylem vessels (embolism) and metabolic and energetic changes depending on environment and genotype.

A portion of water transported through the xylem to leaves is used to transport through phloem to roots organic substances. The water in xylem and mesophyll cell walls move in phloem endings due to osmotic gradient that occurs due to accumulation of sugars and other organic compounds formed in photosynthesis. An inevitable effect of increasing the concentration of moisture is insufficient internal aqueous environment and damages the phloem and xylem transport [17]. This event reflects on the coordination processes of delivery assimilate from leaves to roots and vice versa, water and minerals from the roots to the leaves. Water flow through the xylem of the root apex moves without energy expenditure, due to the use of energy gradient of chemical potential of water between rhizosphere and atmosphere which reaches considerable. Volumetric flow of water from a prolonged drought pauses and water supply plant organs in such conditions is achieved only through symplast transport. In terms of equalizing chemical potential of water in rhizosphere and plant atmosphere consumes considerable amounts of energy to compensate for lack of external driving force of water flow. According to the theory gradients transfer of water through the plant occurs under the free energy gradient. The xylem cell walls and moving water after water potential gradient: from a less negative $\Psi \mathrm{w}$ to a more negative. In symplast active transfer of water is directed against gradient $\Psi \mathrm{w}$. Also suggests that high gradient of $\Psi \mathrm{w}$ of leaves and roots, which occurs in early drought when stomata are still partially open, induce accelerated growth and deepening its root system in the deeper soil horizons. This is one of primary side protection droughtresistant plants, causes plant to adapt to conditions of low humidity. Gradients change status of water plant level is conditioned by the fact that the plant's sensitivity to strain water regime is different.

Generating vascular embolism as could be because extinction gradient in leaf $\Psi \mathrm{w}$ [18]. The central idea developed in this paper is that water potential gradient is generated not only by force of suction sweating and increase activity extinction and increasing cell volume. According to these ideas water potential gradient serves as the driving force for transfer of water and contributes to the extinction of cell through water attraction. In shoot, under favourable conditions, water translocation occurs mostly through xylem and is determined not only by the suction force of swatting and pulsating rhythmic oscillations of micro pressure potential parenchymal cells (is deemed that pulsing cells is a potential mechanism that regulates water and creates local gradients to the flow of water upward) [19]. However, droughts, conditioning xylem embolism vessels, reduce water apoplast transport and increased consumption of energy and metabolites for transport them through symplast active or trans cellular route. In these conditions water transport is carried from cell to cell and transfer the apoplast is small [20]. Most of the water flow passes through the trans cellular pathway including transport through plasmodesma and trans membrane transport. Plasmodesms formed from plasma membrane to endoplasmic reticulum membrane desmotubula are able to pass into other area endo membranes which ensure the integrity of membrane plant tissue. It is assumed that permeability of plasmodesms can be actively controlled account sphincter ATP-dependent operation located in narrow spaces inside plasmodesms [21], and / or rapid and reversible. The main barrier in the way of trans cellular water are membranes. Water transfers them "in the cell-to-cell" and setting her loss is provided at the membrane due to operation of water channel protein (aquaporins) [22]. Aquaporins activity is regulated by aquaporin-genes, or their closing-opening by phosphorylation involving cytosolic $\mathrm{Ca}^{2+}$ or $\mathrm{H}^{+}$. Aquaporins membrane proteins are present in all living plants, which form a complex permeable to water. In drought conditions aquaporins activity is critical in regulating hydraulic conductivity [23] (structures inspection status of water, particularly during drought by changing the hydraulic conductivity). With moisture deficit aquaporins are phosphorylated to a lesser extent and permeability of membranes for water is reduced. Drought resistant plants to maintain its ownership to root hydraulic conductivity at satisfactory level. Restoring hydraulic conductivity tolerant plants coincides with increased activity or abundance aquaporins the cell plasmalemma. Therefore, functional integrity of disturbance caused by an imbalance in absorption of water supply and consumption conditions of drought can be caused not only by disturbances in intensity and water absorption capacity of root system, and hydraulic conductivity of plant. This in turn depends on permeability of cell membrane proteins transporting controlled water activity (aquaporins) and long distance water transport by specialized tissues [24]. Saturation deficit, caused by water stress (drought) advanced hydraulic conductivity results in inhibition of plants with serious consequences on process of water delivery vegetative and generative organs of plant, including apical meristems. These disorders lead to disruption of functional integrity of negative impact on plant growth and development as a whole plant.

\section{Final Remarks}

In summary: coordinating the shoot and root activity and maintaining the functional integrity of the organism level is achieved by different mechanisms. Translocation of carbohydrates from shoot to roots and water and mineral nutrients from the roots to the shoot are balanced according to the source and exporting plant needs. Hydrostatic pressure and plant hormones act as messengers between the two bodies and regulates not only increased the shoot versus root growth, and ensure self-regulation aqueous internal environment by regulating stomatal status and water absorption. Water is not only the primary factor regulating hormonal activity, and enzyme and therefore the metabolic activity. Water is the most effective plant materials contribute to the growth and maintenance of functional integrity. In this context required new knowledge about the mechanisms of coordination and integration of the basic functions of plant resistance and productivity.

\section{References}

1. Sadeghi A, Tonazzini A, Popova L, Mazzolai B (2014) A novel growing device inspired by plant root soil penetration behaviors. PLoS One 9: e90139.

2. van Veen $\mathrm{H}$, Mustroph A, Barding GA, Vergeer-van Eijk M, WelschenEvertman RA, et al. (2013) Two Rumex species from contrasting hydrological niches regulate flooding tolerance through distinct mechanisms. Plant Cell 25 $4691-4707$

3. Couso LL, FernÃ $\tilde{A}_{i n d e z}$ RJ (2012) Phenotypic plasticity as an index of drought tolerance in three Patagonian steppe grasses. Ann Bot 110: 849-857.

4. Gourdji SM, Mathews KL, Reynolds M, Crossa J, Lobell DB (2012) An assessment of wheat yield sensitivity and breeding gains in hot environments. Proc Biol Sci 280: 20122190

5. Mir RR, Zaman-Allah M, Sreenivasulu N, Trethowan R, Varshney RK (2012) Integrated genomics, physiology and breeding approaches for improving drought tolerance in crops. Theor Appl Genet 125: 625-645.

6. Ismail A, Riemann M, Nick P (2012) The jasmonate pathway mediates salt tolerance in grapevines. J Exp Bot 63: 2127-2139. 
7. Anderson JT, Mitchell-Olds T (2011) Ecological genetics and genomics of plant defenses: Evidence and approaches. Funct Ecol 25: 312-324.

8. Yadav A, Singh AL, Rai GK, Singh M (2013) Assessment of molecular diversity in chickpea (Cicer arietinum L.) rhizobia and structural analysis of 16S rDNA sequences from Mesorhizobium ciceri. Pol J Microbiol 62: 253-262.

9. Tian DQ, Pan XY, Yu YM, Wang WY, Zhang F, et al. (2013) De novo characterization of the Anthurium transcriptome and analysis of its digital gene expression under cold stress. BMC Genomics 14: 827.

10. Trivedi DK, Bhatt H, Pal RK, Tuteja R, Garg B, et al. (2013) Structure of RNA interacting cyclophilin A-like protein from Piriformospora indica that provides salinity-stress tolerance in plants. Sci Rep 3: 3001.

11. Champigny MJ, Sung WW, Catana V, Salwan R, Summers PS, et al. (2013) RNA-Seq effectively monitors gene expression in Eutrema salsugineum plants growing in an extreme natural habitat and in controlled growth cabinet conditions. BMC Genomics 14: 578.

12. Zhou XF, Jin YH, Yoo CY, Lin XL, Kim WY, et al. (2013) CYCLIN H;1 regulates drought stress responses and blue light-induced stomatal opening by inhibiting reactive oxygen species accumulation in Arabidopsis. Plant Physiol 162: 1030 1041.

13. Hu X, Wu X, Li C, Lu M, Liu T, et al. (2012) Abscisic acid refines the synthesis of chloroplast proteins in maize (Zea mays) in response to drought and light. PLoS One 7: e49500.

14. Bahrun A, Jensen CR, Asch F, Mogensen VO (2002) Drought-induced changes in xylem $\mathrm{pH}$, ionic composition, and $\mathrm{ABA}$ concentration act as early signals in field-grown maize (Zea mays L.). J Exp Bot 53: 251-263.

15. Smith CM, Desai M, Land ES, Perera IY (2013) A role for lipid-mediated signaling in plant gravitropism. Am J Bot 100: 153-160.
16. Yoneyama K, Awad AA, Xie X, Yoneyama K, Takeuchi Y (2010) Strigolactones as germination stimulants for root parasitic plants. Plant Cell Physiol 51: 10951103.

17. Butnariu M, Butu A (2015) The Effects on Plants by the Insecticides Obtained from Petroleum By-Products, In book Petroleum Engineering Vols. I \& II, Vol. 2: Petrochemical, Petroleum Engg./Chemical Technology, Studium Press LLC USA.

18. Savaldi-Goldstein S, Chory J (2008) Growth coordination and the shoot epidermis. Curr Opin Plant Biol 11: 42-48.

19. von Arx M (2013) Floral humidity and other indicators of energy rewards in pollination biology. Commun Integr Biol 6: e22750.

20. Samardakiewicz S, KrzesÅ,owska M, Bilski H, Bartosiewicz R, WoÅny A (2012) Is callose a barrier for lead ions entering Lemna minor L. root cells? Protoplasma 249: 347-351.

21. J Maule A, Gaudioso-Pedraza R, Benitez-Alfonso Y (2013) Callose deposition and symplastic connectivity are regulated prior to lateral root emergence. Commun Integr Biol 6: e26531.

22. Evkaikina AI, Romanova MA, Voitsekhovskaja OV (2014) Evolutionary aspects of non-cell-autonomous regulation in vascular plants: structural background and models to study. Front Plant Sci 5: 31.

23. Cabrita P, Thorpe M, Huber G (2013) Hydrodynamics of steady state phloem transport with radial leakage of solute. Front Plant Sci 4: 531

24. Carluccio AV, Zicca S, Stavolone L (2014) Hitching a ride on vesicles: cauliflower mosaic virus movement protein trafficking in the endomembrane system. Plant Physiol 164: 1261-1270. 